\title{
Dementia: A Situation for Concern
}

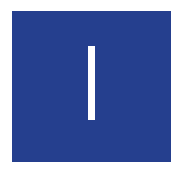
$\mathrm{n}$ this final issue of World Health and Population, the editors have elected to focus on issues related to dementia in elderly persons - and the demands caring for them places on health services delivery. In many parts of the world, aging populations are already causing a strain on health system budgets. Growing numbers of dementia patients further increase the load. The number of people living with dementia worldwide is currently at 50 million and will be approximately three times that number by 2050 (WHO 2019).

It is not just health services systems that feel the burden of caring for dementia patients. Care is often delivered by family members and other informal caregivers, who report feeling isolated and over-burdened (WHO 2019). WHO further reports that dementia has a significant physical, psychological, social and economic impact, not only on people with dementia, but also on their carers, families and the society at large (WHO 2019).

Clearly, the situation is cause for concern. The editors of World Health and Population have chosen to concentrate on three themes related to dementia care: strategies for dementia care; leadership in dementia and new models of care. The intent is to give readers an overview of each area - sharing ideas and policies that have been proven through lived experience.

\section{Strategies for Dementia Care}

The issue opens with a focus on strategies for dementia care that address the needs of patients and their families. Morton-Chang et al. (2016) start from the premise that most older persons living with dementia (PLWD) would prefer to age at home. Too often, however, PLWD end up in residential long-term care (LTC) or in hospital beds - a costly and sometimes damaging solution. The authors explore the "state of the art" in community-based care for PLWD, highlighting the importance of early and ongoing intervention. Drawing on results from a "balance of care" study they conducted earlier, the authors explain that with few viable communitybased care options, PLWD can quickly "default" to institutional care. In the final section, the authors draw from national and international experience to identify the key strategic pillars to guide action towards a community-based dementia care strategy.

Responding to this proposed strategy, Nies (2016) describes the challenge of dealing with increasing numbers of PLWDs and describes potential solutions from the European and, in particular, Dutch perspectives. He concludes with discussion of strategies, required stakeholders, and what conditions need to be in place to arrive at the desired outcomes. 
The third paper in this section focusses on achieving the goals of dementia plans through the lens of evidence-based implementation strategies. Hacker Teper and colleagues (2019) conducted a rapid review of provincial and national dementia plans from high-income countries, and reviewed studies on implementation strategies for dementia care. They advance seven key implementation strategies that may be useful for future dementia care reform.

\section{Leadership in Dementia Care}

Next, attention turns to leadership in dementia care, with specific emphasis on mitigating moral distress. Spenceley et al. (2017) report that while the majority of dementia patients live in the community, residential facility care by nursing providers is a common part of the dementia journey in most countries. The authors share findings from a two-year study of moral distress as experienced by nursing caregivers of residents with dementia in residential care settings in a Western Canadian province. The findings relate to strategies to reduce moral distress in this caregiving group, with a particular focus on the role of supportive and responsive leadership.

\section{New Models of Dementia Care}

The final section in this issue showcases a series of articles that display models of care proven to improve health outcomes, reduce costs and enhance the experience of dementia patients and their caregivers.

Puxty and colleagues (2012) developed a project aimed at facilitating improvements in outcomes for longterm care residents through the provision of knowledge-to-practice and quality improvement resources by trained facilitators. Point-of-care staff reported improved communication and collaboration, improved use of scope of practice and implementation of best practice knowledge. Overall, participating long-term care homes demonstrated an enhanced capacity for common care issues of the elderly (pneumonia, falls, bacteriuria and behavioural and psychological symptoms of dementia) and the ability to effectively engage in quality improvement processes with efficient and effective use of healthcare resources.

Primary care collaborative memory clinics (PCCMCs) address existing challenges in dementia care by building capacity to meet the needs of persons living with dementia within primary care. Lee et al. (2019) describe the strategic implementation of the PCCMC care model in two regions within Ontario. Subsequent interviews and qualitative analysis generated five major themes: (1) earlier identification of dementia and intervention; (2) increased capacity for dementia care within primary care; (3) better patient and caregiver experience with care; (4) improved continuity, integration and coordination and improved care; and (5) system efficiencies. The authors conclude that strategic, regional implementation of PCCMCs provides a significant opportunity to support better integrated and coordinated dementia care.

The following paper looks at specialized seniors' clinics which are an integrated network of six outpatient clinics in British Columbia's Fraser Health Authority that utilize interprofessional teams to provide comprehensive geriatric assessments and care planning for frail older adults. As Kadowaki et al. (2014) explain, the clinics provided approximately 19,000 appointments in 2012/13, and clients and primary care physicians were highly satisfied with the model. The authors outline the SSC model and provide reflection on its development, implementation and standardization processes. 
The issue concludes with a discussion on using technology to assist with transitional care for persons living with dementia. According to Ritchie et al. (2017) transitions between hospital and community are particularly challenging for vulnerable adults experiencing behavioural and psychological symptoms (BPSD) of dementia. Too often, miscommunication results in triggering a recurrence of disruptive behaviours leading to frustration of staff and families. The described project involved improving transitions using an electronic-based care plan on a 23-bed geriatric dementia unit in a mental health hospital. "My Dementia

\section{References}

Hacker Teper,M., C. Godard-Sebillotte and I. Vedel. 2019. Achieving the Goals of Dementia Plans: A Review of Evidence-Informed Implementation Strategies. Healthcare Policy 14(4): 10-20 doi.10.12927/hcpol.2019.25860.

Kadowaki,L., H. Chow, S. Metcalfe and K. Friesen. 2014. Innovative Model of Interprofessional Geriatric Consultation: Specialized Seniors Clinics. Healthcare Quarterly 17(3): 55-60. doi:10.12927/hcq.2014.24021.

Lee, L., L.M. Hillier, K. Lumley-Leger, F. J. Molnar, D. Newton, L. Stirling and K. Milne. 2019. Key Lessons Learned in the Strategic Implementation of the Primary Care Collaborative Memory Clinic Model: A Tale of Two Regions. Healthcare Policy 15(1): 53-69. doi:10.12927/hcpol.2019.25938.

Morton-Chang, F., A.P. Williams, W. Berta and A. Laporte. 2016. Towards a CommunityBased Dementia Care Strategy: How do We Get There from Here? Healthcare Papers 16(2): 8-32. doi:10.12927/hcpap.2017.25006.

Nies, H. 2016. The Journey towards Community-Based Dementia Care: The Destination, Roadmap, Guide, Tour Group and The Conditions. Healthcare Papers 16(2): 57-63. doi:10.12927/hcpap.2017.25001.
Careplan" is an interprofessional care plan that was developed in the electronic medical record (EMR) to enhance communication of information between healthcare providers when patients are being discharged to the community. It is written from the patient's perspective in collaboration with the family and interprofessional team. It describes strategies to manage behavioural challenges along with standardized tools to objectively monitor progress. This care planning will help to support transition of knowledge between hospital and community.

- The Editors

Puxty, J., R. A. Brander, S. Murphy and V. Byrnes. 2012. Promoting Quality Improvement in Long-Term Care: A Multi-Site Collaboration to Improve Outcomes with Pneumonia, Falls, Bacteriuria and Behavioural Issues in Dementia. Healthcare Quarterly 15(2):70-74. doi:10.12927/ hcq.2012.22910.

Ritchie, K., A. Duff-Woskosky and S. Kipping. 2017. Mending the Cracks: A Case Study in Using Technology to Assist with Transitional Care for Persons with Dementia. Nursing Leadership 30(3): 54-62. doi:10.12927/ cjnl.2018.25385.

Spenceley, S., S. Caspar and E. Pijl. 2017. Mitigating Moral Distress in Dementia Care: Implications for Leaders in the Residential Care Sector. Nursing Leadership 30(4): 45-59. doi:10.12927/cjnl.2017.2544.

World Health Organization (WHO). 2019. Dementia. Accessed December 16, 2019. <https://www.who.int/news-room/fact-sheets/ detail/dementia>. 